\title{
Different strategies for cancer treatment: Mathematical modelling
}

\author{
O.G. Isaeva* and V.A. Osipov ${ }^{1}$ \\ Bogoliubov Laboratory of Theoretical Physics, Joint Institute for Nuclear Research, Dubna, \\ Moscow Region, Russia
}

(Received 1 August 2007; final version received 10 September 2008)

\begin{abstract}
We formulate and analyse a mathematical model describing immune response to avascular tumour under the influence of immunotherapy and chemotherapy and their combinations as well as vaccine treatments. The effect of vaccine therapy is considered as a parametric perturbation of the model. In the case of a weak immune response, neither immunotherapy nor chemotherapy is found to cause tumour regression to a small size, which would be below the clinically detectable threshold. Numerical simulations show that the efficiency of vaccine therapy depends on both the tumour size and the condition of immune system as well as on the response of the organism to vaccination. In particular, we found that vaccine therapy becomes more effective when used without time delay from a prescribed date of vaccination after surgery and is ineffective without preliminary treatment. For a strong immune response, our model predicts the tumour remission under vaccine therapy. Our study of successive chemo/immuno, immuno/chemo and concurrent chemoimmunotherapy shows that the chemo/immuno sequence is more effective while concurrent chemoimmunotherapy is more sparing.
\end{abstract}

Keywords: anti-tumour immune response; mathematical modelling; chemotherapy; immunotherapy; vaccine therapy

\section{Introduction}

Some modern trends in treatment of cancer are based on the ability of certain forms of tumours to stimulate immune response. The fact that the immune system plays an important role in fighting cancer has been verified both in laboratorial and clinical experiments [15,34]. An inclusion of immune component in mathematical models of tumour growth has been shown to reflect clinically observed phenomena such as uncontrolled growth of tumour, tumour dormancy and oscillations in tumour size $[1,8,11,12,16,24,25,44,47]$. A similar tumour behaviour was also predicted in our recent ODE model [22] with the interleukine-2 (IL-2) taken into account.

The goal of immunotherapy is to enhance the anti-tumour resistance of an organism and improve the immune system condition. There are known three main categories of immunotherapy: immune response modifiers (cytokines), monoclonal antibodies (MA) and vaccines [40]. Such immune modifiers as IL-2, interferon- $\alpha$ (IFN- $\alpha)$ as well as tumour necrosis factor- $\alpha$ (TNF- $\alpha$ ) are already widely used in cancer immunotherapy $[4,5,19,20,41,48]$. An important problem is to choose the correct schedule for using chemotherapy in combination with IL-2 and IFN- $\alpha$ therapy. For example, a series of sequential phase II trials based on integrating of IL-2 and IFN- $\alpha$ with the CVD (cisplatin, vinblastine and dacarbazine) regimen shows that chemotherapy followed immediately by immunotherapy is more effective for treatment of human metastatic melanoma than their reverse sequence [4,5]. It was also observed that concurrent chemoimmunotherapy is

*Corresponding author. Email: issaeva@theor.jinr.ru 
almost as effective as chemo/immune sequence when immunotherapy is administered right after the CVD. At the same time, the concurrent chemoimmunotherapy is found to be less toxic than the sequential regimens [5]. MA are used in both diagnostics and therapy of cancer. This follows from ability of MA to recognize tumour antigens on a surface of tumour cells. As a result, MA can deliver both anti-tumour drugs and radioactive isotopes exactly to the malignant cells [27,37]. In spite of the fact that cancer vaccines are still under experimental investigations, the existent clinical trials clearly show that they can improve immune response to certain forms of cancer $[40,50]$. Most cancer vaccines consist of living tumour cells and their lysis products while some of them contain tumour-derived proteins, peptides and gangliosides [42]. For instance, an experimental vaccine for malignant melanoma consists of four melanoma peptides restricted by HLA-A1, A2, A3 and HLA-DR and includes IL-2 and granulocyte macrophage colony stimulating factor (GM-CSF) as adjuvants. It was found that this vaccination is able to stimulate tumour regression in some cases [45,46]. The observed toxicity of this vaccination is connected with low doses of IL-2. These findings stimulated our interest to consider within our model the effects of combination immune and chemotherapy treatments as well as vaccine therapy.

One of the first attempts to consider effects of immunotherapy within an appropriate ODE model was made by Kirschner and Panetta [24]. They study immunotherapy based on the use of IL-2 together with adoptive cellular immunotherapy (ACI) by introducing in dynamical equations terms describing external inflow of both IL-2 and cultured immune cells. More recently, de Pillis and Radunskaya have proposed the kinetic model of anti-tumour immune response where individual equations were suggested for the description of mechanisms of natural immunological defence presented by NK-cells and specific immune response presented by CD8 + T cells $[11,12]$. Notice that unlike [24] they do not consider a natural dynamics of IL-2. In the framework of this model, the effects of chemotherapy, immunotherapy, their combined influence, as well as the vaccine therapy were considered [12].

It is interesting to mention a recent paper by Arciero et al. [2] where a novel treatment strategy known as small interfering RNA (siRNA) therapy was considered in the framework of the model proposed in Ref. [24]. This treatment suppresses TGF- $\beta$ production by targeting the mRNA codes for TGF- $\beta$, thereby reducing the presence and effect of TGF- $\beta$ in tumour cells. The model predicts conditions under which siRNA treatment can be successful in transformation of TGF- $\beta$ producing tumours to either non-producing or producing a small value of TGF- $\beta$ tumours, that is to a nonimmune evading state.

In recent years, the importance of spatial aspects of tumour-immune dynamics was demonstrated [30-32,35,36]. For example, in Ref. [31,32] the tumour cell distributions that are quasi-stationary in time and heterogeneous in space were studied within the PDE model by Matzavinos and Chaplain based on the ODE model by Kuznetsov et al. [25]. It was found that depending on model parameters this reaction-diffusion-chemotaxis system is able to simulate the well-documented phenomenon of cancer dormancy, as well as tumour invasion which is presented in the form of a standard travelling wave. The interesting PDE model of non-specific immune response was developed by Owen and Sherrat $[35,36]$. The important conclusion of this model is that macrophages are unable to prevent tumour growth. Nevertheless, significant effects on the form of the tumour were predicted, including the formation of spatial patterns. The model demonstrates the existence of travelling wave solutions connecting the normal tissue and tumour steady states corresponding to a growing tumour. When macrophage chemotaxis is included, these patterns can in some cases bifurcate to give irregular spatiotemporal oscillations.

Finally, let us mention the hybrid cellular automata-PDE modelling approach which combines continuous PDEs for chemical quantities and a discrete cell-based description for biological cell species with phenomenologically sourced probabilities for cell dynamics [30]. This approach allows one to consider both temporal and two-dimensional spatial evolution of the system. 
Numerical simulations include spherical tumour growth, stable and unstable oscillatory tumour growth, satellitosis and tumour infiltration by immune cells.

Our model of immune response to early (avascular) tumour growth is based on the mechanism of intercellular cytokine mediated interaction in cellular immune response proposed by Wagner et al. [49] which was modified by taking into account co-stimulatory factors (see, e.g. Ref. [28,39]). Generally, it consists of seven ordinary differential equations. To simplify analysis, we reduced the model to three equations incorporating the most important modern concepts of tumour-immune dynamics including the influence of IL-2 dynamics (see Ref. [22] for details). Notice also that we do not consider the spatial migration of cell populations that is of most importance for modelling of angiogenesis (vascular growth), invasion and metastasis (see, e.g. Ref. [7]).

In this paper, we extend our model [22] to describe chemo- and immunotherapy effects. The outline of the paper is as follows. First of all, a mathematical model of tumour-immune dynamics under the influence of both immunotherapy with IL-2 and IFN- $\alpha$ and chemotherapy is formulated in Section 2. In Section 3, we perform a steady state analysis of the model. The results of numerical studies are presented in Section 4 for four different cases: chemotherapy alone, IL-2 alone, IL-2 plus IFN- $\alpha$, and a combination of chemotherapy and immunotherapy (IL-2 therapy). The effects of vaccine therapy are considered in the absence of chemotherapy and immunotherapy. Section 5 is devoted to conclusions and discussion.

\section{Mathematical model}

The system of Equations (1)-(5) describe the most important components of tumour-immune dynamics in the presence of treatment components. Namely, we consider five populations: tumour cells $(T)$, CTL $(L)$, IL-2 $\left(I_{2}\right)$, chemotherapeutic drug $(C)$, and IFN- $\alpha(I)$.

$$
\begin{gathered}
\frac{\mathrm{d} T}{\mathrm{~d} t}=-a T \ln \frac{b T}{a}-c(I) T L-M_{T}\left(I_{2}\right)\left(1-\mathrm{e}^{-C}\right) T, \\
\frac{\mathrm{d} L}{\mathrm{~d} t}=d+e L I_{2}-f L-M_{L}\left(I_{2}\right)\left(1-\mathrm{e}^{-C}\right) L, \\
\frac{\mathrm{d} I_{2}}{\mathrm{~d} t}=V_{I_{2}}(t)+\frac{g T}{T+l}-j L I_{2}-k T I_{2}, \\
\frac{\mathrm{d} C}{\mathrm{~d} t}=V_{C}(t)-p C \\
\frac{\mathrm{d} I}{\mathrm{~d} t}=V_{I}(t)-q I .
\end{gathered}
$$

The tumour growth is described by the Gompertzian law (the first term in (1)). The destruction of tumour cells by CTL is presented by the second term in (1). It is supposed that the destruction rate is proportional to the number of tumour cells and CTL populations. In (2), $d$ characterizes the steady inflow of CTL into the tumour site. Second and third terms in (2) describe CTL proliferation in response to the IL-2 action and CTL death rate, correspondingly. In (3)-(5), $V_{i}\left(i=I_{2}, C, I\right)$ describes the external influxes of IL-2, chemotherapeutic drug and IFN- $\alpha$, respectively. Since therapy is assigned to a certain schedule, these influxes are taken to be time-dependent. IL-2 production in (3) is described by hyperbola (the second term), 
which allows us to take into account a limitation in the stimulation of the immune system by the growing tumour. At small $T$ the growth rate is nearly linear in tumour size while for big tumour $(T \gg l)$ it tends to a maximum constant value $g$. The parameter $l$ influences the IL-2 production rate. The smaller the value of $l$, the quicker the IL-2 production rate achieves its maximum value $g$. Notice that $g$ characterizes the degree of expression of the antigen plus major histocompatibility complexes class II (AG-MHC-II) on the APC surfaces, i.e. the antigen presentation. The probability of activation (provoking the IL-2 production) of helper T cell precursor by the APC increases with the antigen presentation. Since IL-2 is a short-distance cytokine, it is suggested that target cells (cytotoxic T lymphocytes) effectively consume IL-2. The consumption rate is presented by the third term in (3). It was found that inhibition of IL-2 results from an accumulation of immune-suppressing substances, prostaglandins. Their number is proportional to the concentration of tumour cells. Prostaglandins suppress the production of IL-2 and can directly destroy its molecules [38]. In (3), the IL-2 destruction rate is described by the fourth term.

The interaction of chemotherapeutic drug with sensitive cells can be described by using of either the Michaelis-Menten kinetics [23] or the exponentially saturating kinetics [12]. Similarly to Ref. [12], we use in Equations (1) and (2) a saturation term $M_{j}\left(I_{2}\right)\left(1-\mathrm{e}^{-C}\right) j$ with $j=T, L$ to describe cell death caused by chemotherapeutic drug. At low concentrations the death rate is nearly linear in drug while at higher concentrations the death rate turns out to be $C$-independent. As was noted in Ref. [12], this behaviour shows a good correlation with existing dose-response curves (see, e.g. Ref. [18]). We assume that $M_{j}$ depends on the IL-2 concentration in the following way: $M_{j}\left(I_{2}\right)=M_{j}^{\text {chemo }}\left(2-\mathrm{e}^{-I_{2} / I_{20}}\right)$. Thus, $M_{j}$ increases with the concentration of IL-2, however, it never exceeds a doubled value of $M_{j}^{\text {chemo }}$ (cell killing by chemotherapy). This is based on the fact that IL-2 can induce the secondary cytokines such as TNF- $\alpha$, which could enhance the anti-tumour effect of cytotoxic chemical agents (see, e.g. Ref. [4]). We also suppose that the model parameter $c$ in (1) depends on the IFN- $\alpha$ concentration as $c(I)=c_{\text {CTL }}\left(2-\mathrm{e}^{-I / I_{0}}\right)$, where $c_{\text {CTL }}$ is a rate of tumour cells inactivation by CTL. This agrees with the fact that IFN- $\alpha$ enhances immune-mediated anti-tumour responses by increasing expression of MHC molecules on tumour cells, thus enhancing their recognition by CTL [48]. Notice that only therapeutic IFN$\alpha$ dynamics is considered within our model.

\subsection{Parameter set}

As is shown in our previous work [22], possible scenarios of tumour-immune dynamics are very sensitive to the choice of the parameters in Equations (1)-(5). In fact the parameter sets vary not only for specific cancer types but also from one individual to another. Our model is based on using of some generalized (most typical) parameters. In order to reflect the individual clinical outcomes, we conditionally divide patients in three groups (see Tables 1 and 2). We assume that tumour has the same histological structure (for instance, melanoma) with equal doubling time and carrying capacity (actually, these characteristics may vary between tumour specimens). Additionally, the lifetime of $\mathrm{CTL}$ is chosen to be the same. On the other hand, the tumour antigen expression $\left(c_{\mathrm{CTL}}\right)$, the strength of the immune response $(e, g, j$ and $k)$, and the reaction to vaccination are taken to be specific for each group. Some values of model parameters were estimated by using the available experimental data. In particular, the human melanoma growth parameters $a$ and $b$ were obtained from the experimental data found in Hu's results on mice trials where human melanoma was tested in severe combined immunodeficient mice [21]. Using the least-squares method, we fitted the experimental curve produced by the data of a control group to Gompertzian curve. The death rate of CTL was estimated using the relation $f=1 / \tau$ where $\tau$ is their known average lifetime. The rate of steady inflow of CTL was calculated from the relation $d=f L_{\text {free }}$ where $L_{\text {free }}$ (the number of CTL capable to recognize melanoma specific antigen in the organism without tumour) was estimated to be about $2.25 \times 10^{7}$ 


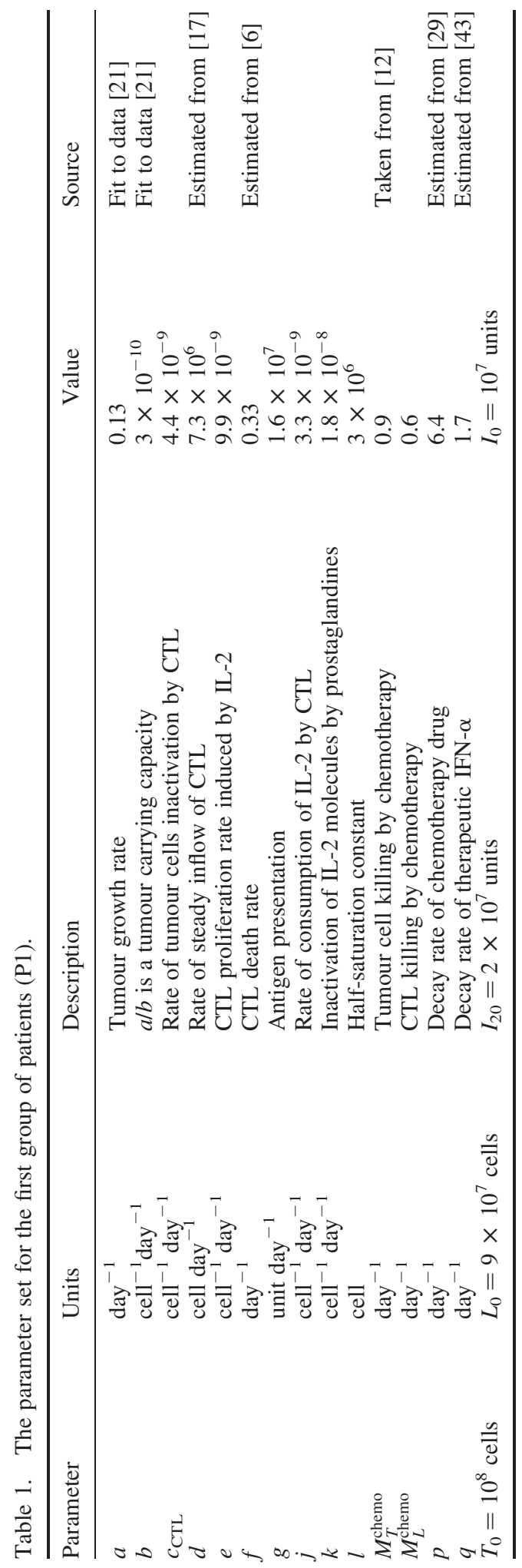


Table 2. The parameter sets for the second (P2) and the third (P3) groups of patients.

\begin{tabular}{lcc}
\hline & \multicolumn{2}{c}{ Value } \\
\cline { 2 - 3 } Parameter & \multicolumn{1}{c}{$\mathrm{P} 2$} & $\mathrm{P} 3$ \\
\hline$a$ & $0.13 \times 10^{-10}$ & 0.13 \\
$b$ & $3 \times 13 \times 10^{-10}$ \\
$c_{\text {CTL }}$ & $3.3 \times 10^{-9}$ & $3.5 \times 10^{-9}$ \\
$d$ & $7.3 \times 10^{6}$ & $7.3 \times 10^{6}$ \\
$e$ & $9.6 \times 10^{-9}$ & $1.0 \times 10^{-8}$ \\
$f$ & 0.33 & 0.33 \\
$g$ & $1.4 \times 10^{7}$ & $2.4 \times 10^{7}$ \\
$j$ & $2.9 \times 10^{-9}$ & $3.7 \times 10^{-9}$ \\
$k$ & $1.5 \times 10^{-8}$ & $2.1 \times 10^{-8}$ \\
$l$ & $3 \times 10^{6}$ & $3 \times 10^{6}$ \\
$M_{T}^{\text {chemo }}$ & 0.9 & 0.9 \\
$M_{L}^{\text {chemo }}$ & 0.6 & 0.6 \\
$p$ & 6.4 & 6.4 \\
$q$ & 1.7 & 1.7 \\
\hline
\end{tabular}

cells using the data for the full number of CD8+ T cells in blood and a percent value of $\mathrm{T}$ cells specific for melanoma antigen [17]. The parameters characterizing the cell death caused by chemotherapeutic influence $M_{T}^{\text {chemo }}$ and $M_{L}^{\text {chemo }}$ are taken from de Pillis's model [12]. The elimination rates for chemotherapeutic drug (dacarbazine) and IFN- $\alpha$ were estimated by using their known half-life times and the relations: $p=\ln 2 / t_{C 1 / 2}$ and $q=\ln 2 / t_{I 1 / 2}$ (see, e.g. Refs. $[29,43]$ ). For the rest of the parameters, we chose values most appropriate to our model. Current medical literature and sensitivity analysis (see Ref. [22]) allow us to conclude that the corresponding interactions are of importance in the description of immune response.

\section{Non-dimensionalization, steady state analysis}

\subsection{Scaling}

For convenience let us introduce dimensionless variables and parameters as follows: $T^{\prime}=T / T_{0}$, $L^{\prime}=L / L_{0}, I_{2}^{\prime}=I_{2} / I_{20}, I^{\prime}=I / I_{0}$ and $t^{\prime}=t / \tau$, where $\tau=f^{-1}$ (days). The values of $T_{0}, L_{0}, I_{20}$ and $I_{0}$ are given in accordance with Ref. [24] and presented in Table 1. Notice that the variable for chemotherapeutic drug, $C$, is given in relative units. The choice of the time-scale factor $\tau$ is based on the fact that the mean lifetime of CTL is about three days and a similar time is needed for the proliferation of CTL and IL-2 production [6,9].

Dropping primes for notational clarity, Equations (1)-(5) take the following form in normalized units:

$$
\begin{gathered}
\frac{\mathrm{d} T}{\mathrm{~d} t}=-h_{1} T \ln \frac{h_{2} T}{h_{1}}-h_{3}\left(2-\mathrm{e}^{-I}\right) T L-m_{1}\left(2-\mathrm{e}^{-I_{2}}\right)\left(1-\mathrm{e}^{-C}\right) T, \\
\frac{\mathrm{d} L}{\mathrm{~d} t}=h_{4}+h_{5} L I_{2}-L-m_{2}\left(2-\mathrm{e}^{-I_{2}}\right)\left(1-\mathrm{e}^{-C}\right) L, \\
\frac{\mathrm{d} I_{2}}{\mathrm{~d} t}=m_{3}(t)+\frac{h_{6} T}{T+h_{9}}-h_{7} L I_{2}-h_{8} T I_{2},
\end{gathered}
$$




$$
\begin{gathered}
\frac{\mathrm{d} C}{\mathrm{~d} t}=m_{4}(t)-m_{5} C, \\
\frac{\mathrm{d} I}{\mathrm{~d} t}=m_{6}(t)-m_{7} I,
\end{gathered}
$$

where $h_{1}=a l f, h_{2}=b T_{0} / f, h_{3}=c_{\mathrm{CTL}} L_{0} / f, m_{1}=M_{T}^{\text {chemo }} / f, h_{4}=d / f L_{0}, h_{5}=e I_{20} / f, m_{2}=M_{L}^{\text {chemo }} / f$, $m_{3}(t)=V_{I_{2}}(t) / f I_{20}, \quad h_{6}=g / f I_{20}, \quad h_{7}=j L_{0} / f, \quad h_{8}=k T_{0} / f, \quad h_{9}=l / T_{0}, \quad m_{4}(t)=V_{C}(t) / f, m_{5}=p / f$, $m_{6}(t)=V_{I}(t) / f I_{0}$ and $m_{7}=q / f$.

\subsection{Steady state analysis}

To perform a steady state analysis we study the system

$$
\begin{gathered}
\frac{\mathrm{d} T}{\mathrm{~d} t}=-h_{1} T \ln \frac{h_{2} T}{h_{1}}-h_{3} T L, \\
\frac{\mathrm{d} L}{\mathrm{~d} t}=h_{4}+h_{5} L I_{2}-L, \\
\frac{\mathrm{d} I_{2}}{\mathrm{~d} t}=\frac{h_{6} T}{T+h_{9}}-h_{7} L I_{2}-h_{8} T I_{2},
\end{gathered}
$$

which follows from (6) to (10) at $V_{i}(t)=0\left(i=I_{2}, C, I\right)$ and $C(0)=I(0)=0$.

A possible way to perform the steady state analysis is to use isoclines. Let us consider the phase plane $T L$, which shows the interactions between two main cell populations: tumour cells and CTL. In this case, the equations for horizontal and vertical isoclines are written as

$$
\begin{gathered}
\left(h_{4}-L\right)\left(T+h_{9}\right)\left(h_{7} L+h_{8} T\right)+h_{5} h_{6} T L=0, \\
L=-\frac{h_{1}}{h_{3}} \ln \frac{h_{2} T}{h_{1}}, \quad T=0 .
\end{gathered}
$$

The fixed points are situated at the intersections of isoclines (14) and (15). Our analysis shows that at any choice of parameters the system of Equations (11)-(13) has the unstable point $\left(0, h_{4}, 0\right)$, which lies at the intersection of isoclines (14) and $T=0$. This means that the regime of full tumour regression is not allowed.

We consider $g$ (characterizing the antigen presentation) as a varying parameter. A bifurcation diagram for the dimensionless parameter $h_{6}$ is presented in Figure 1 where the function $h_{6}(T)$ is obtained by substitution of $L$ from (15) into (14). As is seen, there are two bifurcation points. Therefore one can distinguish three main dynamical regimes. The region I $\left(h_{6}<h_{6 \min }\right)$ characterizes the weak immune response. The systems (11)-(13) have two fixed points: a saddle point $\left(0, h_{4}, 0\right)$ and an improper node $\left(T_{3}, L_{3}, I_{23}\right)$. This means that under a deficiency in the production of IL-2, the population of tumour cells is able to escape from the immune response. The tumour grows and the immune system becomes suppressed. In the region II ( $\left.h_{6 \min }<h_{6}<h_{6 \max }\right)$, which we associate with the strong immune response, there appear two additional fixed points: a stable spiral $\left(T_{1}, L_{1}, I_{21}\right)$ and a saddle $\left(T_{2}, L_{2}, I_{22}\right)$. Therefore different regimes can exist depending on the initial conditions. First, when initial CTL population size is sufficiently large to reduce a tumour population, the tumour regresses up to a small fixed size where the dynamical equilibrium between tumour and immune system is reached. In this case, the tumour manifests itself via the 


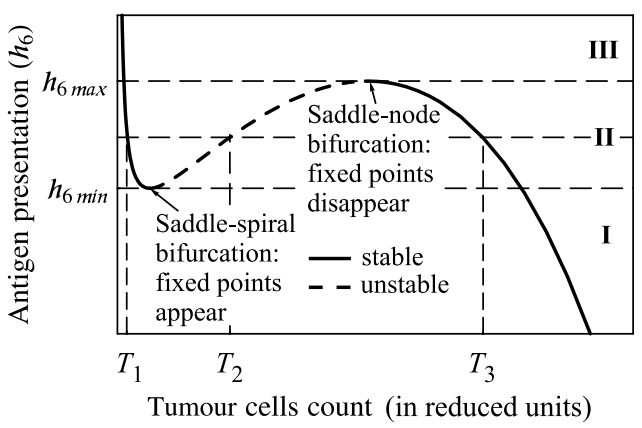

Figure 1. A bifurcation diagram varying the antigen presentation $\left(h_{6}\right)$. For $h_{6}<h_{6 \min }$ there is only one steady state - improper node (region I). When $h_{6 \min }<h_{6}<h_{6 \max }$ there are two stable steady states improper node and spiral node as well as an unstable (saddle) point (region II). For $h_{6}>h_{6 \max }$ only one steady state, the spiral node remains (region III).

excited immune system. Second regime appears when initial number of CTL is not large enough to drive the system at the dynamical equilibrium point $\left(T_{1}, L_{1}, I_{21}\right)$, which is a stable spiral. Thus, the tumour grows to a highest possible size, which is defined for the tumour population being in conditions of restricted feeding. The dynamical equilibrium between the tumour and the immune system is reached at the fixed point $\left(T_{3}, L_{3}, I_{23}\right)$ that is an improper node. Finally, in the region III $\left(h_{6}>h_{6 \max }\right)$ the fixed points $\left(T_{2}, L_{2}, I_{22}\right)$ and $\left(T_{3}, L_{3}, I_{23}\right)$ disappear. As a result, there are two fixed points: a saddle point $\left(0, h_{4}, 0\right)$ and a stable spiral $\left(T_{1}, L_{1}, I_{21}\right)$. In this case, a decrease in tumour size is found when the equilibrium between the tumour and the immune system is established. The region III is associated with the dormant tumour when the immune system is able to handle the tumour size.

\section{Numerical experiments}

In this section, we study the effects of chemotherapy alone, IL-2 alone, IL-2 plus IFN- $\alpha$ therapy, regimens of sequential chemoimmunotherapy as well as vaccine therapy for three groups of patients. Two of them (P1 and P2) generate weak immune responses to the tumour while the third one (P3) generates a strong immune response. The group P2 is characterized by a lower antigen expression in comparison with $\mathrm{P} 1$ and, correspondingly, exhibits a weaker immune response (see Tables 1 and 2). At the stage II of malignant melanoma, both chemotherapy and immunotherapy are usually administered after surgical treatment. Therefore, the initial tumour size is assumed to take a hypothetical value of $T(0) \sim 8 \times 10^{6}$ cells. When we study the effects of treatment administered without preliminary surgery, the initial tumour size is assumed to take a hypothetical value of $3 \times 10^{7}$ cells. In subsections $4.1-4.6$, we will consider the first group of patients (P1).

\subsection{Chemotherapy}

Let us test a treatment approach which employs nine pulsed doses of chemotherapy, each dose represented by setting $V_{C}(t)=1$ in (4) for a day, and given once every 5 days (Figure 2(d)). As is seen from Figure 2(a), a regression is not observed and the tumour population grows. The number of tumour cells oscillates in time as a result of pulsed character of dosing. Tumour growth rate is found to decrease in comparison with the case without treatment. This is completely due to chemotherapeutic influence because the CTL dynamics is slightly affected by chemotherapy (see Figure 2(b)). A possible reason is that an increase in CTL proliferation caused by increasing IL-2 
(a)

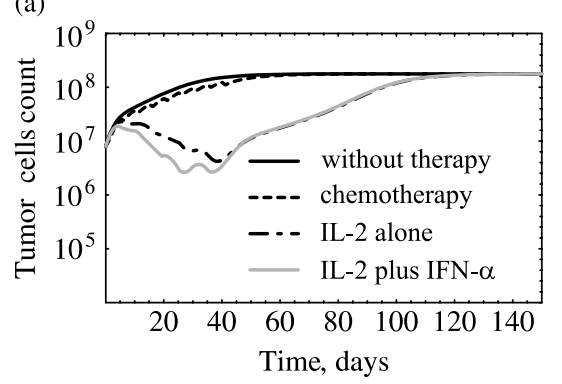

(c)

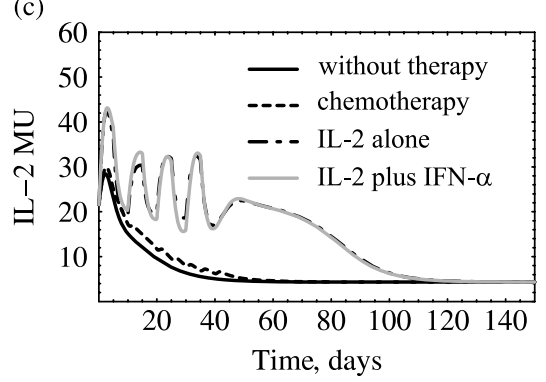

(b)

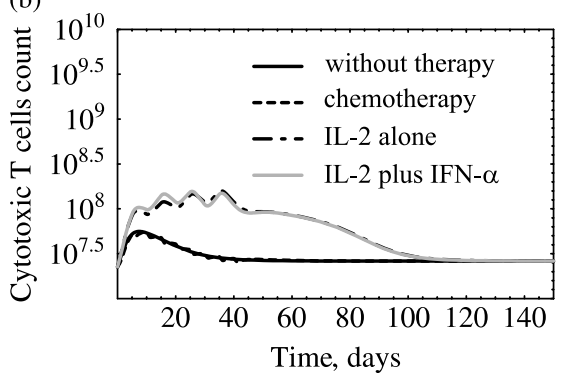

(d)
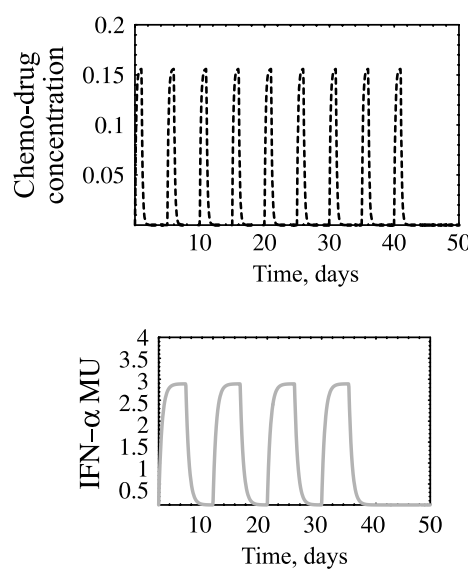

Figure 2. Human data, group P1. Effects of chemo-, IL-2 and IL-2 plus IFN- $\alpha$ therapies on tumour and immune response dynamics. (a) Tumour cells, (b) cytotoxic T cells and (c) IL-2 versus time. (d) Shows drug administration pattern: nine doses, strength $V_{C}(t)=1,1$ day per dose on a 5 days cycle, and IFN- $\alpha$ administration pattern: four doses, strength $V_{I}(t)=5 \mathrm{MU} /$ day, 4 days per dose on a 10 days cycle. IL-2 is administered with four doses of strength $V_{I_{2}}(t)=10 \mathrm{MU} /$ day, 4 days per dose on a 10 days cycle. Initial conditions: $8 \times 10^{6}$ tumour cells, $2.25 \times 10^{7}$ cytotoxic T lymphocytes, $2.4 \times 10^{7} \mathrm{IL}-2$ units.

concentration is compensated by death of CTL under the action of chemo-drug. Thus, our study shows that chemotherapy results in stunted tumour growth. In particular, at our choice of parameters the tumour achieves its dangerous size about 10 days later than in the absence of the therapy.

\subsection{Immunotherapy}

\subsubsection{IL-2 alone}

The following regimen of the IL-2 alone therapy is supposed: four pulsed doses of IL-2, each is equal to $10 \mathrm{MU} /$ day for 4 days, and administered every 10 days. As is seen from Figure 2(a), there is a tumour remission with the duration of about 40 days. At the same time full tumour regression is not observed. Indeed, as IL-2 concentration grows, the CTL population is also increased approximately by a factor of 7 in 40 days (see Figure 2(b)). However, approximately 10 days after treatment cessation the IL-2 concentration decreases (see Figure 2(c)). Accordingly, the CTL population also regresses and, as a result, the tumour growth revives. Thus, this course of treatment leads to a temporary remission only (for $1-1.5$ months in our case). 


\subsubsection{IL-2 plus IFN- $\alpha$}

Let us consider a combined course of the immunotherapy, when IL- 2 and IFN- $\alpha$ are given simultaneously. The dose administration pattern for IL-2 is considered to be the same as in the previous subsection. Together with IL-2 the IFN- $\alpha$ at the dose $5 \mathrm{MU} /$ day for 4 days in a 10-day cycle is administered (Figure 2(d)). As is shown in Figure 2(a), there is a substantial decrease in the number of the tumour cells during the cure. The tumour remission becomes more pronounced in comparison with the previous case although the regression time is almost the same.

Thus, our study shows that immunotherapy is more effective in the remission time of the tumour as compared with chemotherapy. As another conclusion, the IL-2 alone therapy should be considered as more sparing treatment in comparison with the case of IL-2+IFN- $\alpha$. Indeed, in spite of better tumour remission for IL-2+IFN- $\alpha$ treatment the IL-2 alone therapy is less toxic.

\subsection{Sequential chemo/immunotherapy}

In the next three subsections, we study the effects of chemotherapy followed immediately by immunotherapy or vice versa, as well as the concurrent chemoimmunotherapy. We consider the following sequential therapy regimen: one pulse of chemotherapy is presented by setting in (4) $V_{C}(t)=1$ per day for 4 days (Figure 3(d)). During the next 4 days, one pulse of IL-2 therapy is

(a)

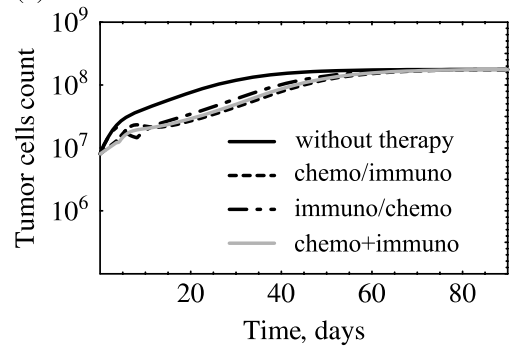

(c)

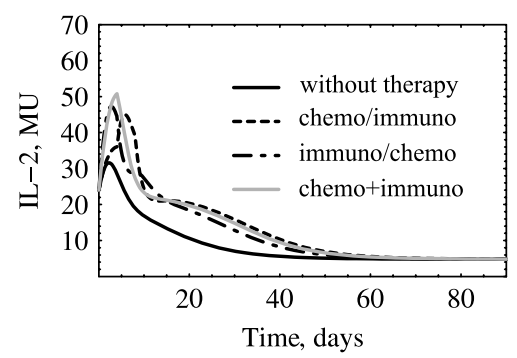

(b)

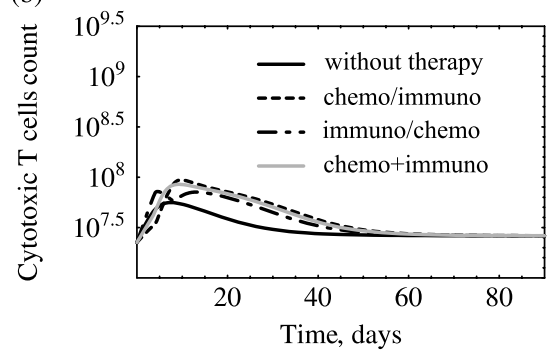

(d)

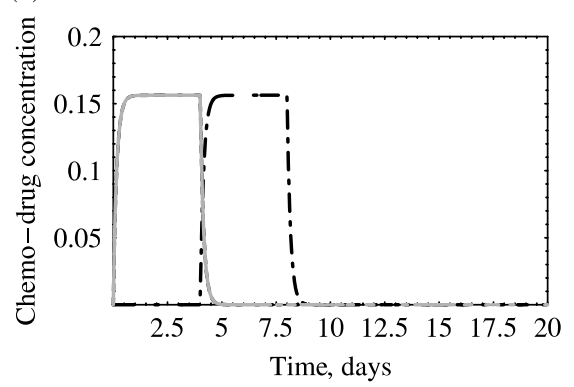

Figure 3. Human data, group P1. Effects of one pulse of chemotherapy followed immediately by one pulse of IL-2-therapy, one pulse of IL-2 therapy followed immediately by one pulse of chemotherapy, and concurrent chemoimmunotherapy. (a) Tumour cells, (b) cytotoxic T cells and (c) IL-2 versus time. (d) Shows drug administration pattern: one pulsed dose of chemotherapy, strength $V_{C}(t)=1$ per day, 4 days per dose for sequential chemo/immunotherapy (dotted line), sequential immuno/chemotherapy (dash-dot line), and concurrent chemoimmunotherapy (grey line). IL-2 administration pattern: one pulsed dose of strength $V_{I_{2}}(t)=10 \mathrm{MU} /$ day, 4 days per dose after chemotherapy (chemo/ immunotherapy sequence) or before chemotherapy (immuno/chemotherapy sequence) and $V_{I_{2}}(t)=7 \mathrm{MU} /$ day for 4 days simultaneously with chemotherapy (concurrent biochemotherapy). Initial conditions: $8 \times 10^{6}$ tumour cells, $2.25 \times 10^{7}$ cytotoxic T lymphocytes, $2.4 \times 10^{7}$ IL-2 units. 
administered in amounts of $V_{I_{2}}(t)=10 \mathrm{MU} /$ day in (3). Figure 3(a) shows the dynamics of tumour cells. As is seen, the chosen regimen of sequential therapy does not lead to the tumour regression. However, a markedly stunted tumour growth is observed (tumour cell population reaches the maximum value about 30 days later in this case). At the initial stage ( $t<8$ days), the tumour growth deceleration is entirely due to chemotherapeutic impact. Furthermore, the tumour cell population slightly decreases. This effect is caused by an increase of the IL-2 concentration during 8 days (see Figure 3(c)), which leads to both a recovery of the CTL number (that has been decreased by chemotherapy) and its following increase (see Figure 3(b)). Later on, the tumour steadily grows and the suppression of the immune functions takes place. Notice that tumour growth rate at this stage is smaller than for $t<8$ days. Thus, although this sequential regimen does not lead to the tumour regression it allows one to delay the tumour growth.

\subsection{Sequential immuno/chemotherapy}

Let us consider the following sequential regimen: one pulse of the IL-2 therapy, which is presented by setting in (3) $V_{I_{2}}(t)=10 \mathrm{MU} /$ day for 4 days. For the next 4 days, one pulse of chemotherapy is administered in dose $V_{C}(t)=1$ in (5) per day for 4 days (Figure 3(d)). The dynamics of tumour cells is shown in Figure 3(a). As is seen, the result of this sequential regimen is worse in comparison with the previous case. Indeed, the IL-2 dosing leads to the increase of its concentration (Figure 3(c)) and, accordingly, to the increase of the CTL number (Figure 3(b)). However, the CTL have not enough time to achieve the magnitude sufficient to slow down the tumour evolution since their growth is abruptly stopped due to chemotherapy (see Figure 3(b)). Nevertheless, at the termination of course of treatment the CTL number again increases due to a sufficiently high concentration of IL-2. As a result, the tumour growth becomes slower reaching a dangerous size twenty days later than in the absence of therapy.

\subsection{The concurrent biochemotherapy}

The regimen of the sequential therapy is chosen to be the following: the chemotherapy in dose $V_{C}(t)=1$ per day and the IL-2 therapy in dose $V_{I_{2}}(t)=7 \mathrm{MU} /$ day are given simultaneously for 4 days. Since the concurrent chemoimmunotherapy is found to be less toxic in comparison with other sequential regimens (see, e.g. Ref. [15]), the dose of the IL-2 is selected to be approximately $3 \times 10^{6}$ units less than in subsections 4.3 and 4.4. As a result, the tumour cell dynamics becomes a little higher in comparison with the first sequential regimen in 4.3 during a period of time that is long enough, except for the initial interval of $(0 ; 10)$ days (see Figure 3(a)). For this period of time, the tumour growth deceleration is more pronounced in comparison with the case of chemo/immuno sequence. Indeed, since chemotherapy and IL-2 therapy are used simultaneously, the tumour cells die under the action of both drug and the immune response recovered by IL-2 therapy. As is seen from Figure 3(b), the dynamics of CTL is similar to that without therapy. For the first 6 days, the IL-2 concentration is higher than in the case of chemo/immunotherapy (Figure 3(c)). Thus, our simulations show that the stronger increase of the IL- 2 concentration prevents the reduction in the CTL number caused by the chemical impact (unlike the first sequential regimen). In turn, for the next 4 days the IL-2 concentration becomes lower as compared with the case of chemo/immunotherapy. Therefore, one can conclude that the concurrent chemoimmunotherapy is more favourable in comparison with the regimen considered in the Subsection 4.3. 


\subsection{Vaccine therapy}

Cancer vaccines are considered as one of promising methods of immunotherapy. Using vaccine allows sensitizing the immune system to the presence of the certain forms of cancer. As a consequence, the immune system will be able to find and lyse tumour cells more effectively. When vaccine appears in the body the anti-tumour lymphocyte formation occurs. The efficacy of the vaccination depends on the following factors: (i) the number of tumour cells and their mitotic activity, (ii) the type of tumour, i.e. its histological structure, antigen structure, the number of HLA-A molecules expressed on the tumour cells and (iii) initial condition of the immune system.

In this subsection, we consider a cancer vaccine consisting of four tumour-derived peptides with an adjuvant (see, e.g. Ref. [33] for a current list of ongoing trials). As long as antigen/adjuvant complexes stimulate immune response to vaccine thereby enhancing immune reaction to patient's tumour cells, the effect of the vaccination can be taken into account through the model parameters. Therefore, in order to simulate vaccine therapy we change the values of four model parameters at the time of vaccination (in a similar manner as in Ref. [12]). The parameters that are sensitive to vaccination can be extracted from the experimental results obtained on mouse vaccine trials by Diefenbach et al. [14]. Namely, we fitted the experimental curves produced by Diefenbach's data to our model and found the parameters that would change to reflect the administration of a therapeutic vaccine. They are $c_{\mathrm{CTL}}$, the rate of inactivation of tumour cells by CTL; $e$, the rate of CTL proliferation induced by IL-2; $g$, the antigen presentation (the probability of interaction between helper T cell precursors and APC) and $j$, the rate of consumption IL-2 by CTL. Finally, to simulate vaccine therapy we alter the corresponding model parameters in the same direction as they change in Diefenbach's murine model [14] (cf. Ref. [12]). As a result, all four parameters $\left(c_{\mathrm{CTL}}, e, g\right.$ and $\left.j\right)$ are found to be increased.

We present here the results for vaccine therapy alone, so that we put $V_{C}(t), V_{I_{2}}(t), V_{I}(t)$ equal to zero as well as $C=I=0$ in (1)-(5). The regimen of vaccination chosen for simulation of vaccine therapy is the following: the cancer vaccine administered once a week during $1-3,5-7$, $13,27,40$ and 53 weeks, respectively [33]. We suppose that the vaccine is effective 83 days after the last injection. This value is not imperative. It seems plausible that this action may last even longer. We assume that at the expiration of this period the system parameters are restored to their initial values. As a result, tumour growth restarts. Therefore revaccination is required to avoid a disease recurrence.

From the above discussion, it is clear that the values of parameters $c_{\mathrm{CTL}}, e, g$ and $j$ will depend on the regimen of vaccination, i.e. on time. In other words, during the vaccine action we increase parameters by a certain percent value (see Table 3). Under these assumptions, the steady-state conditions for P1 become changed in such a way that the system of Equations (11)-(13) passes to the region II on the bifurcation diagram (see Figures 1 and 4). Remember that in this region treatment outcome markedly depends on the initial tumour size and the immune system conditions. Figure 5 shows the results for two courses of the vaccination: the first one was administered

Table 3. The percent increase of the parameters sensitive to the vaccine.

\begin{tabular}{lcc}
\hline & & Increase (in \%) \\
\cline { 2 - 4 } Parameter & P1 & P2, P3 \\
\hline$c_{\text {CTL }}$ & 20 & 10 \\
$g$ & 15 & 20 \\
$j$ & 20 & 30 \\
\hline
\end{tabular}




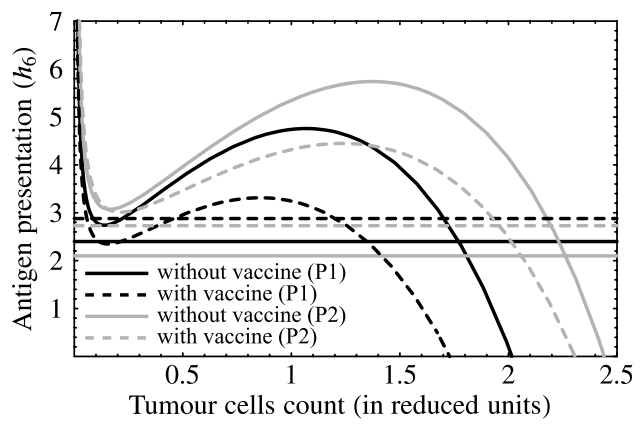

Figure 4. Bifurcation diagrams showing the effect of vaccine therapy on anti-tumour immune response dynamics for P1 and P2.

(a)

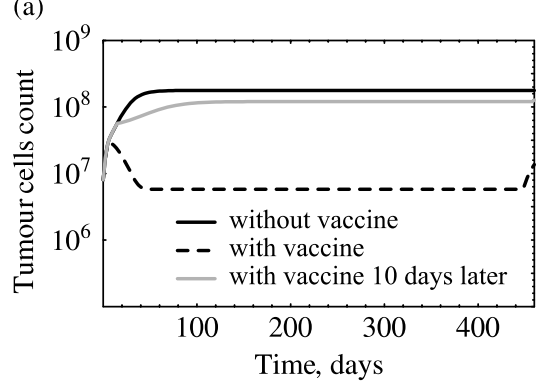

(b)

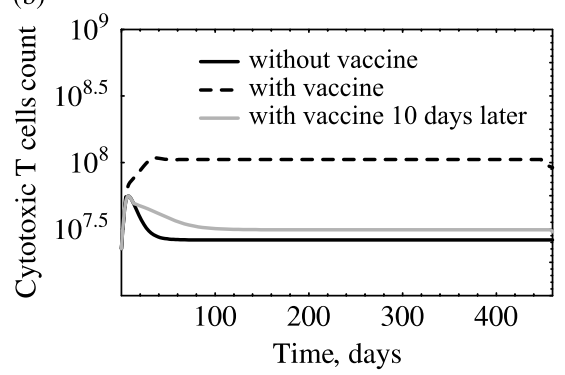

(c)

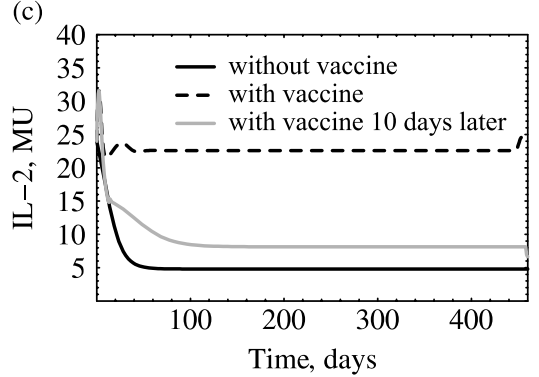

Figure 5. Human data, group P1. Effects of vaccine administered after surgery without delay and with delay for 10 days. (a) Tumour cells, (b) cytotoxic T cells and (c) IL-2 versus time. Initial conditions: $8 \times 10^{6}$ tumour cells, $2.25 \times 10^{7}$ cytotoxic T lymphocytes, $2.4 \times 10^{7} \mathrm{IL}-2$ units.

without delay while the second one was administered 10 days later, when tumour cell population has reached a sufficiently large value to escape the immune response (Figure 5(a)-(c)).

Let us first analyse the behaviour of the system under the vaccine administered after surgery. For therapy without delay, the initial number of tumour cells is enough to induce the immune response. As is seen from Figure 5(c), the IL-2 concentration grows and, consequently, the CTL number is increased. The integral curves tend to the stable spiral point and the long tumour remission is observed (Figure 5(a)). Assumed 10-day delay is simulated by a time displacement $t \rightarrow t+10$. In this case, the tumour has time to reach a sufficiently large size and both the IL-2 concentration and CTL number are decreasing (Figure 5(b),(c)). The integral curves tend to the improper node, which means progressive tumour. The simulations show that the earlier the vaccination is administered the more effective it is for the cancer treatment. 
(a)

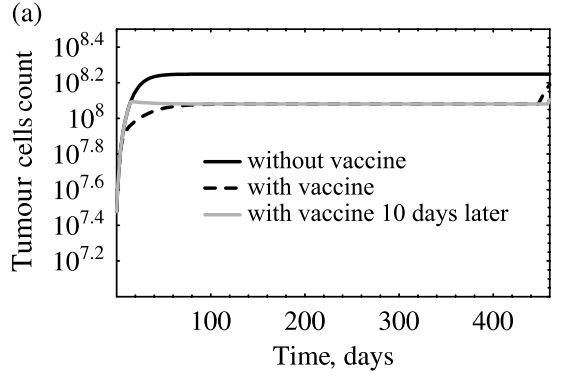

(b)

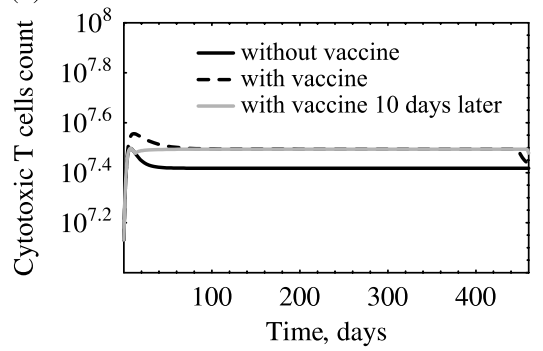

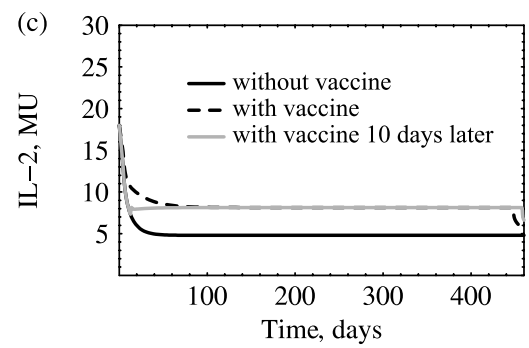

Figure 6. Human data, group P1. Effects of vaccine administered without previous treatment and with delay for next 10 days. (a) Tumour cells, (b) cytotoxic T cells and (c) IL-2 versus time. Initial conditions: $3 \times 10^{7}$ tumour cells, $1.35 \times 10^{7}$ cytotoxic T lymphocytes, $1.8 \times 10^{7} \mathrm{IL}-2$ units.

Let us simulate the vaccine administered without previous surgery. Figure 6(a) shows that even for the therapy without delay the tumour regression does not occur and only some stunted tumour growth with lower saturation level is observed in comparison with the case without therapy. One can suppose that the saturation level without therapy corresponds to a dangerous tumour size in stage II of malignant process. Then the lower saturation level with vaccination may be considered as a steady state of a patient during the vaccine action (Figure 6(a)). In the case of 10-day delay, the tumour size almost reaches the therapeutic saturation level (Figure 6(a)). As is seen, the vaccine-mediated enhancement of the immune response prevents tumour growth to reach a dangerous size. Namely, after 15 days of growth the tumour curve goes slightly down and tends to the therapeutic saturation level. This does not mean, however, that a delay in the vaccination is not dangerous. In fact, as mentioned above, we do not take into account the angiogenesis, which begins at certain size of the tumour and provokes its further explosion $[2,7]$. In other words, the existence of the saturation level does not imply the termination of the tumour growth. Figure 6(b),(c) shows dynamics of CTL and IL-2, respectively.

\subsection{Comparison with second and third groups of patients}

Table 4 summarizes the main findings of subsections 4.1-4.6 for the first group of patients as well as presents the results for two other groups. Let us compare three groups of patients. According to Table 4, for the second group of patients the IL-2 alone and IL-2 plus IFN- $\alpha$ therapies result in slower tumour expansion as compared to chemotherapy. After 6 weeks of the IL-2 alone therapy, the tumour volume reaches almost the same value as in the case of chemotherapy. Results of chemo/immuno regimen are found to be similar to immuno/chemo and concurrent chemoimmunotherapy. This markedly differs from the first group where the increase of tumour size for chemo/immune sequence is smaller in comparison with the reverse sequence. 
Table 4. Effects of different treatments for three groups of patients (the therapies for P1 and P2 are administered after surgery, and for P3 without previous treatment).

\begin{tabular}{|c|c|c|c|c|c|c|c|c|c|c|}
\hline & \multicolumn{2}{|r|}{$\mathrm{P} 1$} & \multicolumn{4}{|c|}{$\mathrm{P} 2$} & \multicolumn{4}{|c|}{ P3 } \\
\hline & $t_{0}$ & $\Delta T$ & $t_{0}$ & $\Delta T$ & $t_{0}$ & $\Delta T$ & $t_{0}$ & $\Delta T$ & $t_{0}$ & $\Delta T$ \\
\hline Chemotherapy & 6 & $\uparrow 13.4$ & 3 & $\uparrow 11.4$ & 6 & $\uparrow 20$ & 3 & $\uparrow 0.1$ & 6 & $\downarrow 0.43$ \\
\hline IL-2 alone & & $\downarrow 0.34$ & & $\uparrow 7.5$ & & $\uparrow 21$ & & $\downarrow 0.82$ & & $\downarrow 0.92$ \\
\hline IL-2 plus IFN- $\alpha$ & & $\downarrow 0.42$ & & $\uparrow \quad 3.9$ & & $\uparrow 10$ & & $\downarrow 0.95$ & & $\downarrow 0.93$ \\
\hline Chemo/immune & & $\uparrow 9.92$ & & $\uparrow 9.3$ & & $\uparrow 23.9$ & & $\downarrow 0.68$ & & $\downarrow 0.88$ \\
\hline Immune/chemo & & $\uparrow 12.8$ & & $\uparrow 10.2$ & & $\uparrow 24.3$ & & $\downarrow 0.48$ & & $\downarrow 0.88$ \\
\hline Chemo + immune & & $\uparrow 10.9$ & & 9.5 & & $\uparrow 24$ & & $\downarrow 0.63$ & & $\downarrow 0.88$ \\
\hline $\begin{array}{l}\text { Vaccine therapy without } 10 \\
\text { day delay }\end{array}$ & & $\downarrow 0.25$ & & $\uparrow 8.92$ & & $\uparrow 18.7$ & & $\downarrow 0.91$ & & $\downarrow 0.91$ \\
\hline
\end{tabular}

The arrow $\uparrow$ means the increase of the tumour size, $\downarrow-$ the decrease of the tumour size. The change of tumour size is presented as $\Delta T=\left(T\left(t_{0}\right)-T(0)\right) / T(0)$. $t_{0}$ - the time after the start of treatment, weeks. For P1 and P2 the initial conditions are $T(0)=8 \times 10^{6}$ cells, $L(0)=2.25 \times 10^{7}$ cells, $I(0)=2.4 \times 10^{7}$ cells, for P3 $-T(0)=3 \times 10^{7}$ cells, $L(0)=3.45 \times 10^{7}$ cells, $I(0)=1.7 \times 10^{7}$ cells.

Therefore, one can conclude that the dependence on the schedule is more pronounced in the first group. As a possible reason, the IL-2 therapy is less effective in the second group. This is a result of lower tumour antigen expression when stimulation of CTL proliferation by IL-2 becomes insufficient for effective recognition of tumour cells. Although the IL-2 plus IFN- $\alpha$ therapy looks more favourable for P2 in comparison with other therapies, one has to bear in mind that the vaccine therapy is less toxic. Let us consider the behaviour of the second group of patients in response to the vaccination in more detail. The increase of corresponding parameters for P2 during the vaccine action is shown in Table 3. As is seen, the suggested values differ from these for P1. Figure 4 shows that in this case the steady-state conditions do not change. This result looks rather unexpected. In fact, it means absence of the positive clinical response despite the fact that the immune reaction to the tumour is taken to be enhanced by the vaccine even better as compared with the first group. Indeed, we have intentionally taken the bigger relative growth of parameters $e, g$ and $j$ for $\mathrm{P} 2$ in comparison with $\mathrm{P} 1$.

Figure 7 shows the results of the vaccination after surgery and Figure 8 shows the case without preliminary treatment. In the first case, using vaccine without delay allows stunting tumour growth and it reaches the therapeutic saturation level in 70 days. Besides, with vaccine the saturation level becomes lower than without therapy. It should be noted that the vaccine administered with 10 days delay is not effective because no deceleration of the tumour growth is observed (Figure 7(a)). The vaccination is ineffective when it is administered without preliminary surgery (Figure $8(\mathrm{a})$ ).

It should be noted that the positive response to the treatment can also be described within our model. In order to show this possibility, we consider the third group of patients (P3 in Table 2). In the absence of treatment, the tumour grows to the dangerous size (see Figure 9). As is seen from Table 4, all of the considered therapeutic regimens result in tumour regression to the small volume that corresponds to the stable fixed point (spiral node) of the system. For sequential regimens the slower decreasing of the tumour volume is found as compared to IL-2 alone, IL-2 plus IFN- $\alpha$ and vaccine therapies. There is more pronounced regression of the tumour size in the cases of chemo/immune sequence and concurrent chemoimmunotherapy in comparison with the immune/chemo sequence. At the same time, we would like to mention more than $50 \%$ decrease of the tumour size that implies the effectiveness of all considered regimens. Thus, our simulations show that after cessation of therapy the tumour regresses. 
(a)

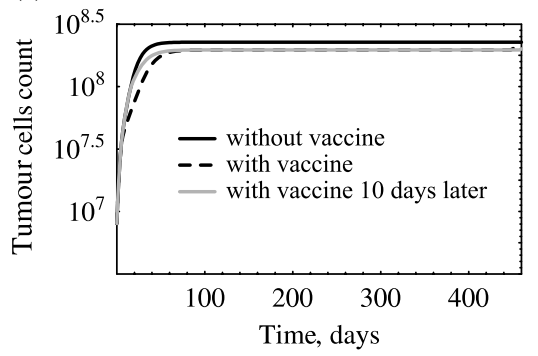

(b)

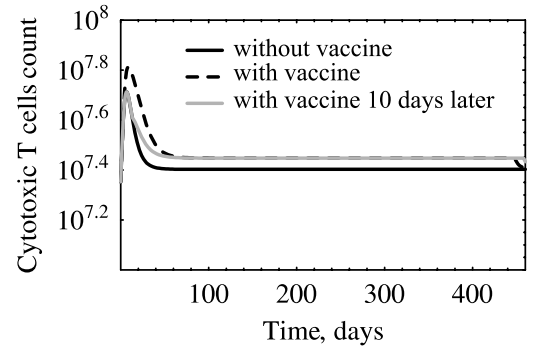

(c)

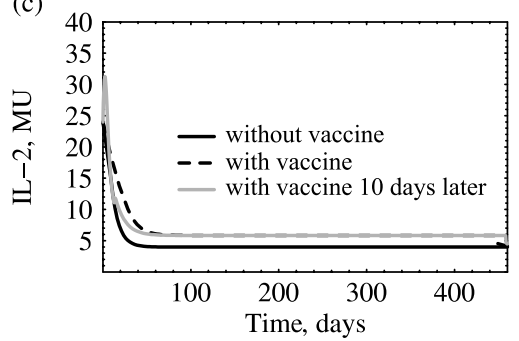

Figure 7. Human data, group P2. Effects of vaccine administered after surgery without delay and with delay for 10 days. (a) Tumour cells, (b) cytotoxic T cells and (c) IL-2 versus time. Initial conditions: $8 \times 10^{6}$ tumour cells, $2.25 \times 10^{7}$ cytotoxic T lymphocytes, $2.4 \times 10^{7} \mathrm{IL}-2$ units.

(a)

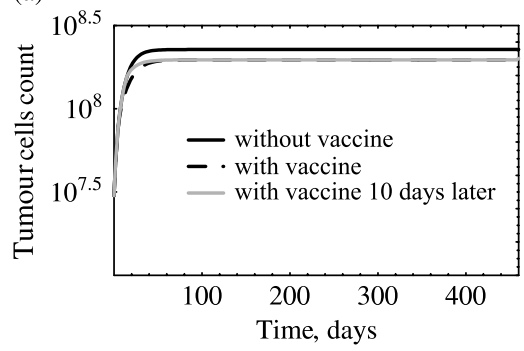

(b)

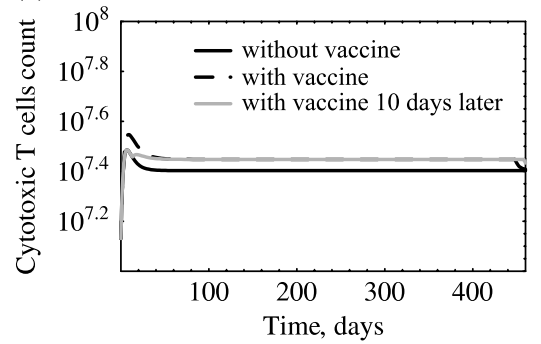

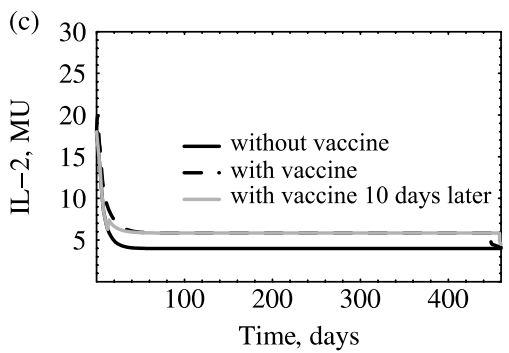

Figure 8. Human data, group P2. Effects of vaccine administered without previous treatment and with delay for next 10 days. (a) Tumour cells, (b) cytotoxic T cells and (c) IL-2 versus time. Initial conditions: $3 \times 10^{7}$ tumour cells, $1.35 \times 10^{7}$ cytotoxic T lymphocytes, $1.8 \times 10^{7}$ IL-2 units. 
(a)

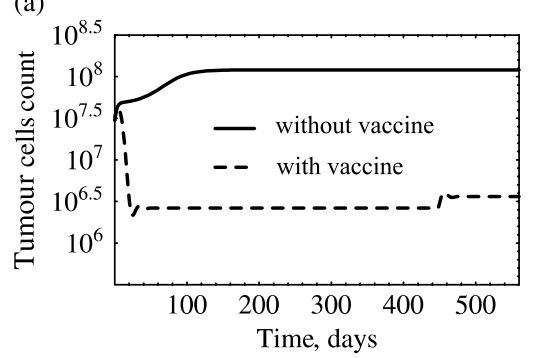

(b)

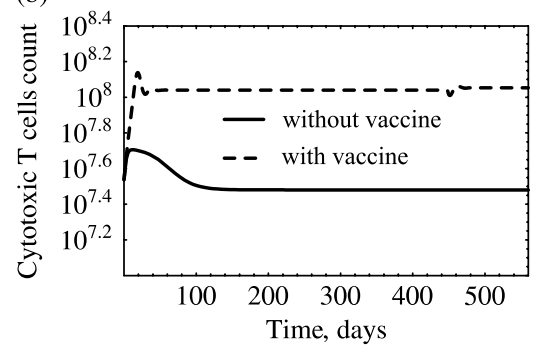

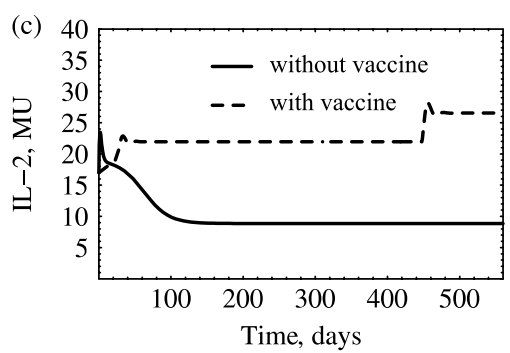

Figure 9. Human data, group P3. Effects of vaccine administered without previous treatment. (a) Tumour cells, (b) cytotoxic T cells and (c) IL-2 versus time. Initial conditions: $3 \times 10^{7}$ tumour cells, $3.45 \times 10^{7}$ cytotoxic T lymphocytes, $1.7 \times 10^{7} \mathrm{IL}-2$ units.

The interesting results are obtained in the case of the vaccine therapy (see Figure 9). We consider the situation when under the vaccine therapy the system passes to the region III in Figure 1 (see Table 3). In this case, the effect of vaccine therapy does not depend on the initial tumour size and the immune system conditions. As a result, the time delay is out of importance (without angiogenesis taken into account). As is seen from Figure 9(a), the tumour cells population decreases to a small size. While after the termination of the vaccine action the tumour regrows, it nevertheless never exceeds the size $T_{1}$ (corresponding to the stable spiral for P3).

\section{Conclusion}

We have studied the effects of different treatment regimens on both the tumour growth and the immune response within the simple ODE model that describes tumour-immune dynamics with chemotherapy and immunotherapy. It is found that the regime of full regression of tumour is not admitted in our model. This conclusion is in agreement with some current clinical observations where recurrences of tumours are observed [26]. The bifurcation diagram for antigen presentation shows three main dynamical regimes. The region I reflects a progressive growth when the tumour is able to escape from the immune response. The region II describes two regimens of disease depending on both the initial tumour size and the condition of immune system: (i) the regression to small tumour when the dynamical equilibrium is established and (ii) a progressive tumour growth to the highest possible size. For the region III the decrease of the tumour size is found when the equilibrium between the tumour and the immune system is established.

In order to describe a possibility of different responses to treatment regimens, patients were conditionally divided in three generalized groups. Each group is characterized by specific tumour antigen expression, the strength of the immune response, and the reaction to vaccination. For patients with a weak immune response the vaccine therapy is found to be the most effective in comparison with other described treatments when used without time delay from a prescribed 
date of vaccination after surgery. This means that using vaccine gives the best results for patients with both small size of tumour and an immune system, which is not suppressed by tumour growth and able to respond to the vaccine. For the first group, the vaccine therapy is shown to be the only possible treatment allowing long tumour remission. Therefore, we note a promising effect of the vaccine treatment to improve immune response for this group. This qualitatively agrees with clinically observed results (see, e.g. Refs. [40,45,46,50]). For the second group, all considered treatments result in progressive growth. However, the vaccine therapy without delay after surgery is expected to be more sparing. We observed that for patients with a strong immune response IL-2 alone, IL-2 plus IFN- $\alpha$ and the sequential chemoimmunotherapy could be used as a reasonable alternative to vaccination.

Our study shows that along with progressive disease the positive clinical responses to the treatment characterized by a long remission of tumour growth are possible. This qualitatively agrees with modern clinical observations. Indeed, clinical trials of chemotherapy and sequential regimens for melanoma showed that along with progressive diseases the partial responses and even complete responses were possible within patient groups [26]. It was also shown in the trials of the vaccine therapy [45] that the CTL response to the vaccine by itself did not guarantee the tumour regression. For instance, for several patients the $\mathrm{T}$ cell response to the vaccine was found to be not strong enough to decrease the tumour size and, as a result, the tumour was progressing. At the same time, the tumour regression was observed for few patients with immune responses to the vaccine. In our model, these observations could be explained by both heterogeneity of the tumour antigen expression and patient-specific characteristics of immune response.

It should be stressed that all these predictions can be valid only for the description of early stages of the tumour growth when the processes of angiogenesis, invasion and metastasis are not of critical importance. As the next step, we plan to extend our simple ODE model to PDE model to include spatial components for distributions of cells and drugs. In this context, the presented analysis is of interest since it gives the underlying kinetics of more general PDE model. Notice also that toxicity of both chemotherapy and immunotherapy requires finding of an optimal treatment protocol. There are known some attempts to examine this problem within appropriate ODE models of tumour-immune dynamics (see e.g. Refs. $[3,10,13]$ ) and it would be interesting to consider it within our model. These studies are now in progress.

\section{Note}

1. Email: osipov@theor.jinr.ru

\section{References}

[1] J.A. Adam and N. Bellomo, A Survey of Models for Tumor-Immune System Dynamics, Birkhäuser, Boston, MA, 1996.

[2] J.C. Arciero, D.E. Kirschner, and T.L. Jackson, A mathematical model of tumor-immune evasion and siRNA treatment, Disc. Cont. Dyn. Syst-B 4 (2004), pp. 39-58.

[3] T. Burden, J. Ernstberger, and K. Renee Fister, Optimal control applied to immunotherapy, Discr. Contin. Dyn. Syst. Ser. B 4(1) (2004), pp. 135-146.

[4] A.C. Buzaid, Strategies for combining chemotherapy and biotherapy in melanoma, Cancer Control 7(2) (2000), pp. 185-197.

[5] A.C. Buzaid and M. Atkins, Practical guidelines for the management of biochemotherapy-related toxicity in melanoma, Clin. Cancer Res. 7 (2001), pp. 2611-2619.

[6] D.L. Chao, M.P. Davenport, S. Forrest, and A.S. Perelson, A stochastic model of cytotoxic T cell responses, J. Theoret. Biol. 228 (2004), pp. 227-240.

[7] M.A.J. Chaplain, Mathematical models in cancer research, in The Cancer Handbook, Chapter 60, Nature Publishing Group, New York, 2003, pp. 937-951. 
[8] R.J. De Boer, P. Hogeweg, F.J. Dullens, R.A. De Weger, and W. Den Otter, Macrophage T lymphocyte interactions in the anti-tumor immune response: A mathematical model, J. Immunol. 134 (1985), pp. 2748-2758.

[9] R.J. De Boer, M. Oprera, R. Antia, K. Murali-Krishna, R. Ahmed, and A.S. Perelson, Recruitment times, proliferation, and apoptosis rates during the CD $+T$ cell Response to lymphocytic choriomeningitis virus, J. Virol. 75 (2001), pp. 10663-10669.

[10] L.G. de Pillis and A. Radunskaya, A mathematical tumor model with immune resistance and drug therapy: An optimal control approach, J. Theoret. Med. 3 (2001), pp. 79-100.

[11] L.G. de Pillis and A.E. Radunskaya, Immune response to tumor invasion, in Computational Fluid and Solid Mechanics, K. Bathe, ed.,Vol. 2, MIT Press, Cambridge, 2003, pp. 1661-1668.

[12] L.G. de Pillis, W. Gu, and A.E. Radunskaya, Mixed immunotherapy and chemotherapy of tumors: Modeling, applications and biological interpretations, J. Theoret. Biol. 238(4) (2006), pp. 841-862.

[13] L.G. de Pillis, W. Gu, K.R. Fister, T. Head, K. Maples, T. Neal, A. Murugan, and K. Yoshida, Chemotherapy for tumors: An analysis of the dynamics and a study of quadratic and linear optimal controls, Math. Biosci. 209 (2007), pp. 292-315.

[14] A. Diefenbach, E.R. Jensen, A.M. Jamieson, and D. Raulet, Rael and H60 ligands of the NKG2D receptor stimulate tumor immunity, Nature 413 (2001), pp. 165-171.

[15] J.D. Farrar, K.H. Katz, J. Windsor, G. Thrush, R.H. Scheuermann, J.W. Uhr, and N.E. Street, Cancer dormancy. VII. A regulatory role for CD $8+T$ cells and IFN-gamma in establishing and maintaining the tumor-dormant state, J. Immunol. 162(5) (1999), pp. 2842-2849.

[16] R. Garay and R. Lefever, A kinetic approach to the immunology of cancer: Stationary states properties of effector-target cell reactions, J. Theoret. Biol. 73 (1978), pp. 417-438.

[17] S. Garbelli, S. Mantovani, B. Palermo, and C. Giachino, Melanocyte-specific, cytotoxic T cell responses in vitiligo: The effective variant of melanoma immunity, Pigment Cell Res. 18 (2005), pp. 234-242.

[18] S.N. Gardner, A mechanistic, predictive model of dose-response curves for cell cycle phase-specific and nonspecific drugs, Cancer Res. 60 (2000), pp. 1417-1425.

[19] B.L. Gause, M. Sznol, W.C. Kopp, J.E. Janik, J.W. Smith II, R.G. Steis, W.J. Urba, W. Sharfman, R.G. Fenton, S.P. Creekmore, et al., Phase I study of subcutaneously administered interleuking-2 in combination with interferon alfa-2a in patients with advanced cancer, J. Clin. Oncol. 14 (1996), pp. 2234-2241.

[20] I. Hara, H. Hotta, N. Sato, H. Eto, S. Arakava, and S. Kamidono, Rejection of mouse renal cell carcinoma elicited by local secretion of interleukin-2, J. Cancer Res. 87 (1996), pp. 724-729.

[21] Z. Hu, Y. Sun, and G. Alan, Targeting tumor vasculature endothelial cells and tumor cells for immunotherapy of human melanoma in a mouse xenograft model, Proc. Natl Acad. USA 96 (1999), pp. 8161-8166.

[22] O.G. Isaeva and V.A. Osipov, Modeling of anti-tumor immune response: Immunocorrective effect of weak centimeter electromagnetic waves, J. CMMM (2008) (to be published). Available at http://ru.arxiv.org/abs/q-bio/0506006.

[23] T.L. Jackson, Vascular tumor growth and treatment: Consequences of polyclonality, competition and dynamic vascular support, J. Math. Biol. 44 (2002), pp. 201-226.

[24] D. Kirschner and J.C. Panetta, Modeling immunotherapy of the tumor-immune interaction, J. Math. Biol. 37 (1998), pp. 235-252.

[25] V.A. Kuznetsov, I.A. Makalkin, M.A. Taylor, and A.S. Perelson, Nonlinear dynamics of immunogenic tumors: Parameter estimation and global bifurcation analysis, Bull. Math. Biol. 56(2) (1994), pp. 295-321.

[26] S.S. Legha, S. Ring, A. Bedikian, C. Plager, O. Eton, A.C. Buzaid, and N. Papadopoulos, Treatment of metastatic melanoma with combined chemotherapy containing cisplatin, vinblastin and dacarbazine (CVD) and biotherapy using interleukin-2 and interferon- $\alpha$, Ann. Oncol. 7 (1996), pp. 827-835.

[27] J. Li, K. Guo, V.W. Koh, J.P. Tang, B.Q. Gan, H. Shi, H.X. Li, and Q. Zeng, Generation of PRL-3and PRL-1-specific monoclonal antibodies as potential diagnostic markers for cancer metastases, Clin. Cancer Res. 11(6) (2005), pp. 2195-2204.

[28] Y. Liu, Y. Ng, and K.O. Lillehei, Cell mediated immunotherapy: A new approach to the treatment of malignant glioma, Cancer Control 10 (2003), pp. 138-147.

[29] T.J. Loo, G.E. Housholder, A.H. Gerulath, P.H. Saunders, and D. Farquhar, Mechanism of action and pharmacology studies with DTIC (NSC-45388), Cancer Treat. Rep. 60 (1976), pp. 149-152.

[30] D.G. Malleta and L.G. de Pillis, A cellular automata model of tumor-immune system interactions, J. Theoret. Biol. 239 (2006), pp. 334-350. 
[31] A. Matzavinos and M.A.J. Chaplain, Travelling-wave analysis of a model of the immune response to cancer, C R Biol. 327(11) (2004), pp. 995-1008.

[32] A. Matzavinos, M.A.J. Chaplain, and V.A. Kuznetsov, Mathematical modelling of the spatiotemporal response of cytotoxic T-lymphocytes to a solid tumour, Math. Med. Biol. 21 (2004), pp. $1-34$.

[33] National Cancer Institute, 2005. National cancer institute clinical trials web site. Available at http:// www.nci.nih.gov/clinicaltrials accessed December 2005.

[34] K.J. O'Byrne, A.G. Dalgleish, M.J. Browning, W.P. Steward, and A.L. Harris, The relationship between angiogenesis and the immune response in carcinogenesis and the progression of malignant disease, Eur. J. Cancer 36 (2000), pp. 151-169.

[35] M.R. Owen and J.A. Sherratt, Pattern formation and spatiotemporal irregularity in a model for macrophage-tumour interactions, J. Theoret. Biol. 189 (1997), pp. 63-80.

[36] —, Mathematical modelling of macrophage dynamics in tumours, Math. Models Methods Appl. Sci. 9 (1999), pp. 513-539.

[37] Z. Qu, G.L. Griffiths, W.A. Wegener, C.H. Chang, S.V. Govindan, I.D. Horak, H.J. Hansen, and D.M. Goldenberg, Development of humanized antibodies as cancer therapeutics, Methods 36(1) (2005), pp. 84-95.

[38] RESAN Scientific Research Enterprise, Scientific research enterprise web site, 2003. Available at http://www.anticancer.net/resan/basis.html\#interleukins.

[39] I. Roitt, J. Brostoff, and D. Male, Immunology, 6th ed., Mosby, London, 2001, 480pp.

[40] E. Rosenbaum and I. Rosenbaum, Everyone's Guide to Cancer Supportive Care: A Comprehensive Handbook for Patients and Their Families, Andrews McMeel Publishing, Riverside, 2005.

[41] S.A. Rosenberg and M.T. Lotze, Cancer immunotherapy using interleukin-2 and interleukin-2activated lymphocytes, Annu. Rev. Immunol. 4 (1986), pp. 681-709.

[42] V. Schirrmacher, V. Umansky, M. Linder, S. Müerköster, and M. Rocha, Models for immunotherapy and Cancer Vaccines, in The Cancer Handbook, Chapter 68, Nature Publishing Group, New York, 2003, pp. 1055-1068.

[43] Shenzhen Neptunus Interlong Bio-Tech Holdings Co., Interlong web site, 2005. Available at http:// www.interlong.com/en/Products.asp.

[44] J. Sherrat and M. Nowak, Oncogenes, anti-oncogenes and the immune response to cancer: A mathematical model, Proc. R. Soc. Lond. B 248 (1992), pp. 261-271.

[45] C.L. Slingluff, Jr., G.R. Petroni, G.V. Yamshchikov, D.L. Barnd, S. Eastham, H. Galavotti, J.W. Patterson, D.H. Deacon, S. Hibbitts, D. Teates, et al., Clinical and immunologic results of randomised phase II trial of vaccination using four melanoma peptides either administered in GM-CSF in adjuvant or pulsed on dendritic cells, J. Clin. Oncol. 21(21) (2003), pp. 4016-4026.

[46] C.L. Slingluff, Jr., G.R. Petroni, G.V. Yamshchikov, S. Hibbitts, W.W. Grosh, K.A. ChianeseBullock, E.A. Bissonette, D.L. Barnd, D.H. Deacon, J.W. Patterson, et al., Immunologic and clinical outcomes of vaccination with a multiepitope melanoma peptide vaccine plus low-dose interleukine-2 administered either concurrently or on a delayed schedule, J. Clin. Oncol. 22(22) (2004), pp. 4474-4485.

[47] N. Stepanova, Course of the immune reaction during the development of a malignant tumor, Biophysics 24 (1980), pp. 917-923.

[48] M. Sznol and T. Davis, Antibodies and recombinant cytokines, in The Cancer Handbook, Chapter 87, Nature Publishing Group, New York, 2003, pp. 1371-1379.

[49] H. Wagner, C. Hardt, K. Heeg, K. Pfizenmaier, W. Solbach, R. Bartlett, H. Stockinger, and M. Rollingoff, $T$-T cell interactions during CTL response: $T$ cell derived helper factor (interleukin 2) as a probe to analyze CTL responsiveness and thymic maturation of CTL progenitors, Immunol. Rev. 51 (1980), pp. 215-255.

[50] C.J. Wheeler, D. Asha, L. Genatao, J.S. Yu, and K.L. Black, Clinical responsiveness of glioblastoma multiforme to chemotherapy after vaccination, Clin. Cancer Res. 10 (2005), pp. 5316-5326. 


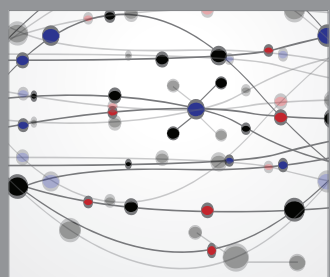

The Scientific World Journal
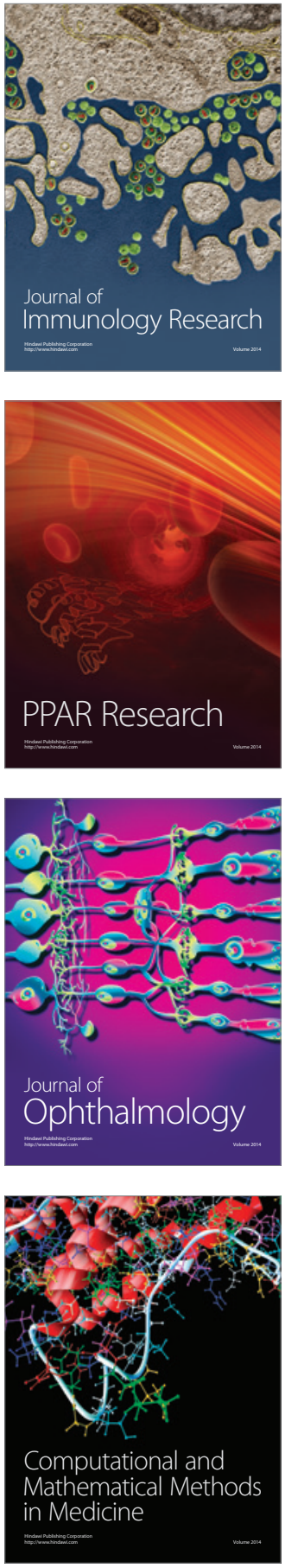

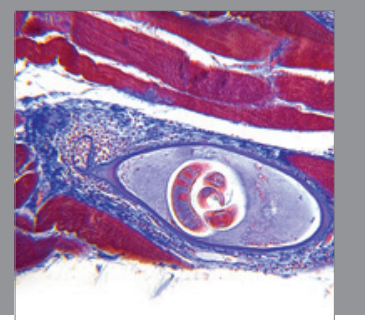

Gastroenterology

Research and Practice
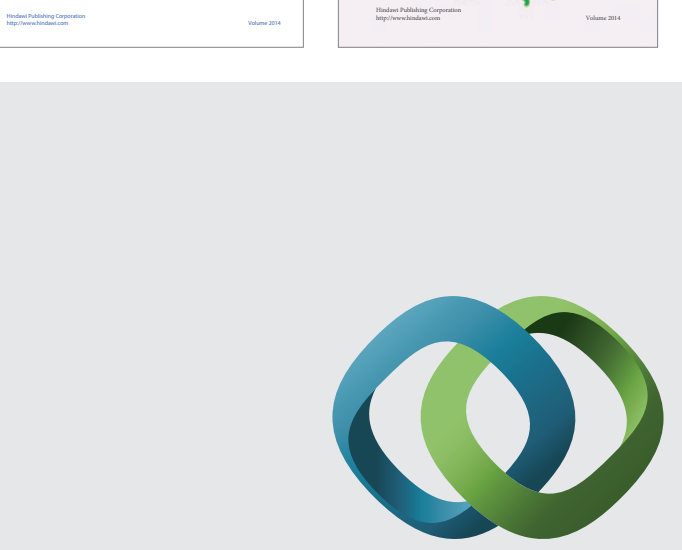

\section{Hindawi}

Submit your manuscripts at

http://www.hindawi.com
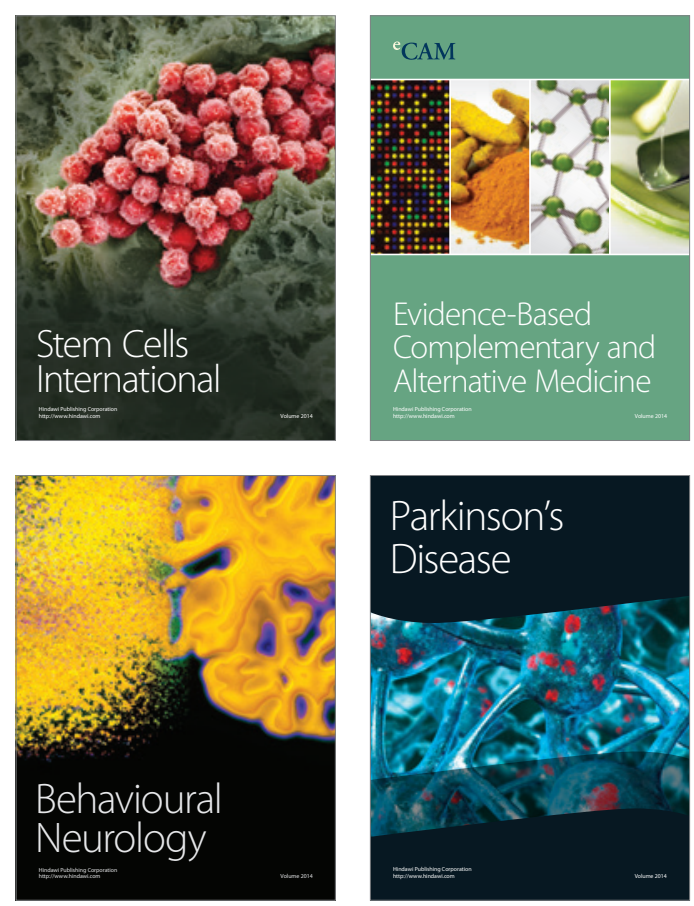

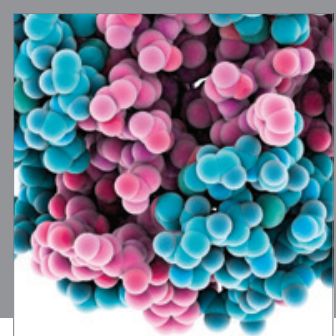

Journal of
Diabetes Research

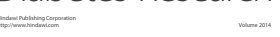

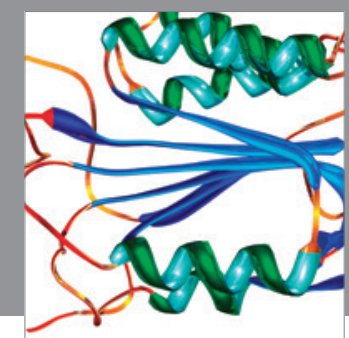

Disease Markers
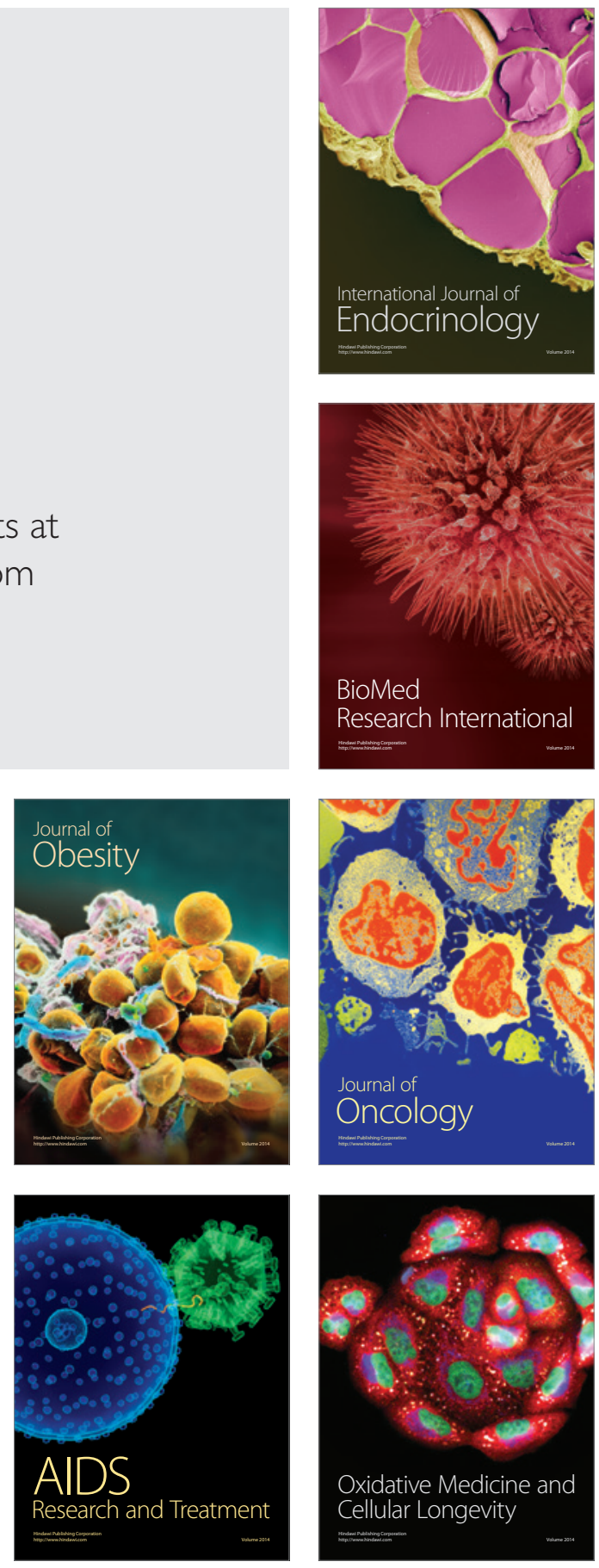